\title{
ON THE STRUCTURE OF THE SCHRÖDINGER PROPAGATOR
}

\author{
ANDREW HASSELL AND JARED WUNSCH
}

\begin{abstract}
We discuss the form of the propagator $U(t)$ for the timedependent Schrödinger equation on an asyptotically Euclidean, or, more generally, asymptotically conic, manifold with no trapped geodesics. In the asymptotically Euclidean case, if $\chi \in \mathcal{C}_{0}^{\infty}$, and with $\mathcal{F}$ denoting Fourier transform, $\mathcal{F} \circ e^{-i r^{2} / 2 t} U(t) \chi$ is a Fourier integral operator for $t \neq 0$. The canonical relation of this operator is a "sojourn relation" associated to the long-time geodesic flow. This description of the propagator follows from its more precise characterization as a "scattering fibered Legendrian," given by the authors in a previous paper and sketched here.

A corollary is a propagation of singularities theorem that permits a complete description of the wavefront set of a solution to the Schrödinger equation, restricted to any fixed nonzero time, in terms of the oscillatory behavior of its initial data. We discuss two examples which illustrate some extremes of this propagation behavior.
\end{abstract}

\section{INTRODUCTION}

Let us consider the Schrödinger initial value problem for a particle moving in curved space with metric $g$

$$
\left(D_{t}+\frac{1}{2} \Delta+V\right) \psi(z, t)=0, \quad \psi(0, \cdot)=\psi_{0},
$$

with $D_{t}=-i \partial_{t}$ and $\Delta$ the nonnegative Laplace-Beltrami operator

$$
-\frac{1}{\sqrt{g}} \partial_{z^{i}} g^{i j} \sqrt{g} \partial_{z^{j}}
$$

and where $V$ is a smooth, real-valued potential function. The solution is given in terms of functional calculus by the formula

$$
\psi(t, \cdot)=e^{-i t H} \psi_{0}, \quad H=\frac{1}{2} \Delta+V,
$$

and the operator $e^{-i t H}$ (or its kernel) is called the propagator, or fundamental solution. On flat $\mathbb{R}^{n}$ with $V=0$, there is an explicit formula for the propagator for (11): its Schwartz kernel is

$$
K(z, w, t)=(2 \pi i t)^{-n / 2} e^{i|z-w|^{2} / 2 t} .
$$

This may also be thought of as the solution of (11) with initial data the delta function at $w$ (the initial value problem makes sense for any tempered distribution as initial data). 
This solution exhibits some peculiar properties, from the point of view of propagation phenomena. Fixing $w=w_{0} \in \mathbb{R}^{n}$, we find that for any $t>0, K\left(t, z, w_{0}\right)$ is smooth. Hence the initial delta-function singularity at $z=w_{0}$ apparently disappears. Additionally, since the propagator is, by self-adjointness of $\Delta$, unitary on $L^{2}$, we can reverse this process: If we take

$$
\psi_{0}=e^{i \lambda|z|^{2} / 2+\xi \cdot z}
$$

as smooth initial data for a solution to (1), then at $t=-\lambda^{-1}$ the solution develops a delta-function singularity, located at $z=-\xi / \lambda$. One can see this either by explicitly convolving with $K(z, w, t)$ and doing a Gaussian integral, or by noting that this initial data is nothing but a multiple of a time-translated version of the fundamental solution.

The upshot, then, is that singularities to solutions of the Schrödinger equation can both appear and disappear. In this paper, we address these phenomena not just on $\mathbb{R}^{n}$, but more generally on curved space. The basic questions are where do singularities go when they disappear, and, conversely, what causes their appearance? (These questions are based on the idea that, in evolution described by a unitary group, things should not "disappear" and "appear," but rather be transformed into something more or less equivalent.) The most powerful tool for answering propagation questions is to understand the kernel of the propagator: a sufficiently precise understanding of it, particularly its asymptotics at infinity, will allow us to answer both questions. We describe a generalization of the formula (2) and an interpretation thereof which enables us to describe the formation and disappearance of singularities. We should emphasize that by a singularity at time $t$ we mean a point in the wavefront set of $\psi(t, \cdot)$ in the sense of Hörmander [8]. We recall that a point in the wavefront set of a distribution is an element of the unit cosphere bundle, $(z, \hat{\zeta})$ with $|\hat{\zeta}|=1$, which intuitively describes an infinitesimal wave located at $z$ with wavefronts normal to $\hat{\zeta}$.

This note is intended as a "user's guide" to the authors' more technical paper [7] which describes both a parametrix construction for the Schrödinger propagator and, as a corollary, a full propagation of singularities theorem answering the two questions posed above.

Much has long been known about the structure of the propagator on $\mathbb{R}^{n}$ with nonzero potentials $V$; indeed a number of rather precise parametrix constructions exist (among others, those of Fujiwara [3], Zelditch [19], Trèves [15, Yajima [17).

On curved space, on the other hand, almost nothing was known about the propagator until comparatively recently. The first variable-coefficient results were those of Kapitanski-Safarov [10], who showed that in the case of a compactly-supported metric perturbation of $\mathbb{R}^{n}$ with no trapped geodesics the kernel of the propagator is smooth for all $t>0$. The same authors subsequently constructed a parametrix 9, albeit without the control at infinity which will turn out to be essential for the purposes at hand. At 
around the same time, Craig-Kappeler-Strauss proved the first result about microlocal regularity of solutions to (11). Loosely speaking, they showed that on asymptotically Euclidean space, if the initial data $\psi_{0}$ is a Schwartz function in a conic microlocal neighborhood of a backward geodesic passing through a point $(z, \hat{\zeta}) \in S^{*} \mathbb{R}^{n}$, then $(z, \hat{\zeta}) \notin \mathrm{WF} \psi(t)$ for all $t>0$. In particular, let $\chi(z)$ denote a cutoff function supported in a positive cone near infinity. Assume that $\chi(z) \psi_{0} \in \mathcal{S}$. Now let $\gamma(s)$ be any geodesic so that $\gamma(s) \in \operatorname{supp} \chi$ for all $s<<0$. We conclude that $\left(\gamma(s), g_{i j}\left(\gamma^{\prime}\right)^{j}(s)\right) \notin$ $\mathrm{WF} \psi(t, z)$ for any fixed $t>0$. Thus a single hypothesis gives regularity along the (co-)tangents to a whole pencil of geodesics emanating from supp $\chi$ for all $t>0$. In Euclidean space, this yields regularity along the tangents to all lines lying in a pencil of directions, and thus constrains the direction in which singularities of $\psi$ can lie.

The second author [16] refined the result of Craig-Kappeler-Strauss by introducing the quadratic wavefront set "at infinity," measuring the quadratic oscillation of a distribution ${ }^{1}$. It is analogous to the scattering wavefront set previously introduced by Melrose [12] to describe linear oscillations. In [16] conditions were given, in terms of the quadratic scattering wavefront set, that constrain not only the directions in which singularities can appear, but also the times at which they can appear. The actual locations, however, remain mysterious from this point of view. Theorems such as these, which determine $\zeta$ and $t$ but not $z$ in the wavefront set $(z, \zeta) \in \mathrm{WF} \psi(t)$ have been called microglobal results 18 .

The quadratic-scattering wavefront set results of [16] have recently been extended, using a rather different set of tools, to the analytic category by Robbiano-Zuily [13; in this setting, even defining the quadratic scattering wavefront set involves an appropriate two-parameter version of the FBI transform, rather than the pseudodifferential methods employed in [12, 16].

The first author thanks the Australian Research Council for its support, through a Fellowship and a Linkage grant, of this research. The second author acknowledges the support of the National Science Foundation, through grants DMS-0100501 and DMS-0323021.

\section{The GEOMETRY}

The results of [7] hold for a rather general class of manifolds with large conic ends, known as scattering manifolds, introduced by Melrose [12. However for simplicity we will restrict ourselves here to a sub-class consisting of asymptotically Euclidean spaces. Let $z$ be a Euclidean coordinate and let

$$
\theta=\frac{z}{|z|}, r=|z|
$$

\footnotetext{
${ }^{1}$ For example, in the case of a distribution $e^{i \phi(z)}$, where $\phi$ is homogeneous of degree two, hence determined by a function $\tilde{\phi}$ on $S^{n-1}$, the quadratic wavefront set is essentially the graph of $\tilde{\phi}$ over the sphere at infinity.
} 
be polar coordinates. We will assume that our metric is smooth and has the form

$$
g=\sum_{i=1}^{n}\left(d z^{i}\right)^{2}+h, \quad h=\frac{m d r^{2}}{r}+\frac{k_{i j}\left(r^{-1}, \theta\right) d z^{i} d z^{j}}{r^{2}} \text { for } r \gg 0,
$$

where $m$ is a constant and $k_{i j}$ is a $\mathcal{C}^{\infty}$ function of its arguments, i.e. has an asymptotic expansion (Taylor series) in descending powers of $r$.

We further make the following crucial nontrapping assumption ${ }^{2}$ for $g$ :

$$
\text { For any geodesic } \gamma(t), \lim _{t \rightarrow \pm \infty} r(\gamma(t))=\infty \text {. }
$$

As for the potential $V$, we assume $V \in \mathcal{C}^{\infty}\left(\mathbb{R}^{n} ; \mathbb{R}\right)$ and for $r \gg 0$,

$$
V=\frac{c}{r}+\frac{\tilde{V}\left(r^{-1}, \theta\right)}{r^{2}}
$$

with $c$ a constant and $\tilde{V}$ a $\mathcal{C}^{\infty}$ function of its arguments. If $m$ in (3) and $c$ in (15) both vanish, then $H$ is said to be short-range, otherwise (gravitational) long-range.

We henceforth denote the Hamiltonian

$$
H=\frac{1}{2} \Delta+V,
$$

hence the propagator is $e^{-i t H}$.

\section{The FORM OF THE PROPAGATOR}

In order to motivate our results about the Schwartz kernel of the propagator, let us re-examine the special form (2) of the Euclidean one. If we introduce polar coordinates in the $w$ variable, we have

$$
K(t, r, \theta, z)=e^{i r^{2} / 2 t}\left(a e^{-i z \cdot \theta r / t}\right)
$$

where $a=(2 \pi i t)^{-n / 2} e^{i w^{2} / 2 t}$. We have factored $K$ into this form for several reasons. First, we are considering $K$ as a function of $r, \theta$ alone, with $t>0$ and $z$ fixed. Hence $a$ is, from this point of view, constant. Second, while $K$ is a rather uninteresting distribution from the point of view of wavefront set, it is certainly not a Schwartz function, owing to its oscillatory behavior at infinity. We have chosen to exhibit this oscillatory behavior by separating out the leading order factor $e^{i r^{2} / 2 t}$, from the milder, plane wave oscillation of $e^{-i z \cdot \theta r / t}$. Recall now that part of our goal in constructing the propagator is to understand the fate of the delta-function singularity launched from the point $z \in \mathbb{R}^{n}$. From this point of view, the leading order term $e^{i r^{2} / 2 t}$ is useless: it retains no information about where the initial delta function lay, and merely records, in its frequency $t^{-1}$, the elapsed time. By contrast, the plane wave oscillation term is of great interest: we can recover $z$ from $e^{-i z \cdot \theta r / t}$. Finally, note that $t$ appears in the phase in a very simple way.

\footnotetext{
${ }^{2}$ It is actually not necessary to make this assumption, but if we do not then the results only apply in the non-trapping part of phase space.
} 
Since the quadratic term is of little interest, we will separate it explicitly in our formula for the propagator. Thus, for $t \neq 0$, and for $H$ short-range, let

$$
W_{t}=e^{-i r^{2} / 2 t} e^{-i t H} .
$$

In the Euclidean case, this is a constant multiple of a plane wave $e^{-i z \cdot \theta r / t}$. Fourier transforming in $w=r \theta$, then, gives a delta distribution $\delta(z-w)$. This turns out to be a rather general phenomenon.

Let $\mathcal{F}$ denote the Fourier transform and let $\chi \in \mathcal{C}_{c}^{\infty}\left(\mathbb{R}^{n}\right)$. One of the main results from [7] can be expressed as

Theorem 1. Suppose $H$ is short-range and $t \neq 0$. Then the operator

$$
\mathcal{F} \circ W_{t} \chi
$$

is a classical Fourier integral operator of order 0 on $\mathbb{R}^{n}$ associated to a canonical transformation of $S^{*} \mathbb{R}^{n}$.

Remark. The point of multiplying on the right by $\chi$ is to localize the right spatial variable to a compact set. Theorem 1 is essentially concerned with the behaviour of the propagator when the left variable approaches infinity. What happens as both variables independently approach infinity is more complicated.

Let $Q$ denote the Fourier integral operator of Theorem 1 and $\gamma$ the contact transformation. Then a standard property of FIOs $[8$ is that $Q$ moves wavefront set according to $\gamma$ :

$$
\mathrm{WF}(Q f) \subset \gamma(\mathrm{WF} f) .
$$

It is now easier to see why the propagator creates and destroys wavefront set: can can write $e^{-i t H} \chi=e^{i r^{2} / 2 t} \mathcal{F}^{-1} Q$. Thus $Q$ moves wavefront set around, but $\mathcal{F}^{-1}$ kills it (if it is compactly supported). So a better way to think of the above theorem is that $W_{t}$ maps wavefront set to "Fourier transformed wavefront set."

In the gravitational long-range case, we need to modify the radial variable slightly. Let

$$
\tilde{r}=r+\frac{m}{2} \log r
$$

and define

$$
W_{t}=e^{-i \tilde{r}^{2} / 2 t} e^{-i t H} .
$$

This modification is familiar in, for example, the structure of generalized eigenfunctions or of Dollard wave operators for the Schrödinger operator with Coulomb potential [2], 14]. Then $\mathcal{F} \circ W_{t} \chi$ is a quasi-classical ${ }^{3}$ Fourier integral operator of order zero.

\footnotetext{
${ }^{3}$ Quasi-classical here means that the symbol $a(x, y, \theta)$ has an expansion that includes $\log$ terms as well as powers of $\theta$.
} 
To understand the canonical relation of Theorem 1 will require understanding the long-time limit of geodesic flow. We begin, however, by clarifying the action of $W_{t}$ itself on singularities of distributions.

\section{Scattering WAVEFront Set}

We have seen that in order to describe the mapping properties of the propagator on wavefront set, it is helpful to keep track of Fourier transformed wavefront set. The wavefront set of a tempered distribution on $\mathbb{R}^{n}$ is a closed subset of $S^{*} \mathbb{R}^{n}$; it is convenient for our purposes to identify this space with the product $\mathbb{R}^{n} \times S^{n-1}$.

Fourier transforming a compactly supported distribution with nonempty wavefront set - a delta-function for instance - yields a smooth function with oscillation at infinity - e.g. a plane wave. In tracking where the singularity has gone under Fourier transform, it is therefore helpful to introduce a wavefront set that measures oscillation at infinity. On $\mathbb{R}^{n}$, we simply use the Fourier transform to do this.

Definition 2. The scattering wavefront set of a distribution $u \in \mathcal{S}^{\prime}\left(\mathbb{R}^{n}\right)$ is the closed subset

given by

$$
\mathrm{WF}_{\mathrm{sc}} u \subset S_{\hat{z}}^{n-1} \times \mathbb{R}_{\zeta}^{n}
$$

$$
(\hat{z}, \zeta) \in \mathrm{WF}_{\mathrm{sc}} u \Longleftrightarrow(\zeta, \hat{z}) \in \operatorname{WF} \mathcal{F}(u)
$$

So, for instance,

$$
\mathrm{WF}_{\mathrm{sc}} e^{i \xi \cdot z}=\left\{(\hat{z}, \xi): \hat{z} \in S^{n-1}\right\}
$$

captures the frequency of plane wave oscillation.

Remark. The scattering wavefront set was introduced by Melrose 12] in the more global context of scattering manifolds, where the definition is more subtle. Melrose's definition encompasses the set defined above, which we think of as a subset of an appropriately scaled cotangent bundle at infinity, and the ordinary wavefront set inside the cosphere bundle, as well as a third component which interpolates between the two, and is a subset, in our noninvariant notation, in $S_{\hat{z}}^{n-1} \times S_{\hat{\zeta}}^{n-1}$ (the cosphere bundle at infinity).

The quadratic-scattering wavefront set of [16] can be defined quite similarly: again a subset of $S^{n-1} \times \mathbb{R}^{n}$, it can be defined by

$$
\mathrm{WF}_{\mathrm{qsc}} u=\mathrm{WF}_{\mathrm{sc}} \tilde{u}
$$

where $\tilde{u}$ is the distribution defined by

$$
\tilde{u}(z)=u(z / \sqrt{|z|}) .
$$

Theorem 11 now implies that $W_{t}=e^{-i r^{2} / 2 t} e^{-i t H}$ maps scattering wavefront set to ordinary wavefront set, and vice versa. In particular, given the non-invariance of the Fourier transform, it is best from a geometrical point of view to think of $W_{t}$ as a "scattering Fourier integral operator" with a wavefront relation interchanging $\mathbb{R}^{n} \times S^{n-1}$ and $S^{n-1} \times \mathbb{R}^{n}$. 


\section{The CANONiCAL RELAtion}

The canonical relation of $W_{t}$ as a scattering FIO is related to the limit of geodesic flow. Given $(z, \hat{\zeta}) \in S^{*} \mathbb{R}^{n} \cong \mathbb{R}^{n} \times S^{n-1}$ we let $\gamma(t)$ be the unit speed geodesic with $\gamma(0)=z,\left(\gamma^{\prime}(0)\right)^{i}=g^{i j} \hat{\zeta}_{j}$, and define (in the short range $\left.\operatorname{case}^{4}\right)$

$$
\begin{aligned}
S_{f}(z, \hat{\zeta}) & =(\theta, \xi) \in S^{n-1} \times \mathbb{R}^{n}, \text { with } \xi=\lambda \theta+\mu, \\
\theta & =\lim _{t \rightarrow \infty} \frac{\gamma(t)}{|\gamma(t)|}, \\
\lambda & =\lim _{t \rightarrow \infty} t-|\gamma(t)| \\
\mu & =\lim _{t \rightarrow \infty}|\gamma(t)|\left(\theta-\frac{\gamma(t)}{|\gamma(t)|}\right) .
\end{aligned}
$$

We also define $S_{b}(z, \hat{\zeta})=-S_{f}(z,-\hat{\zeta})$. These are the forward and backward sojourn relations, respectively. We interpret the components of these maps as follows: $\theta$ is the asymptotic direction of the geodesic, $\lambda$ is the "sojourn time," a measure of how long the geodesic lingers in the finite part of $\mathbb{R}^{n}$ before heading off to infinity, and $\mu$ measures the angle of contact of the geodesic with the sphere at infinity, or, equivalently, distinguishes different geodesics among the pencil of all geodesics with the same asymptotic direction.

We may endow $\mathbb{R}^{n} \times S^{n-1}$ with a contact structure using the canonical one-form on $S^{*} \mathbb{R}^{n}$; by switching coordinates, as in the definition of scattering wavefront set, we may thus endow $S^{n-1} \times \mathbb{R}^{n}$ with a contact structure.

Proposition 3. The maps

$$
S_{f}, S_{b}: \mathbb{R}^{n} \times S^{n-1} \rightarrow S^{n-1} \times \mathbb{R}^{n}
$$

are contact diffeomorphisms.

Hence $S_{f}$ and $S_{b}$ are eligible to be quantized to scattering Fourier integral operators.

Theorem 4. The canonical relation of $W_{t}$ is $|t|^{-1} S_{f}$ for $t<0$ and $|t|^{-1} S_{b}$ for $t>0$, with the scaling acting in the fiber variable.

\section{A sketch of the Construction}

In [7] a result considerably more detailed than Theorem 1 is proven: it is shown that $W_{t}$ is a scattering-fibered Legendrian distribution in both space and time variables simultaneously. Legendrian distributions are a class of distributions introduced by Melrose-Zworski in scattering theory 11]; they are given by oscillatory integrals and on $\mathbb{R}^{n}$ are essentially the same as Fourier transforms of Hörmander's Lagrangian distributions. The

\footnotetext{
${ }^{4}$ In the long range case there is a logarithmic divergence in $\lim t-|\gamma(t)|$ which needs to be removed. We omit the details.
} 
scattering-fibered Legendrians are a refinement introduced by Hassell-Vasy [5, 6]. This finer characterization of $W_{t}$ amounts to the statement that it is a linear combination of distributions of the form

$$
t^{-\frac{n}{2}-\frac{k}{2}} \int_{U \Subset \mathbb{R}^{k}} a\left(t, r^{-1}, \theta, z, v\right) e^{i \phi\left(r^{-1}, \theta, z, v\right) r / t} d v
$$

with $a$ and $\phi$ smooth in the short-range case (and having expansions in the system $r^{-n}(\log r)^{k}$ for $k \leq n$ in general), and with $\phi$ satisfying a nondegeneracy condition; see [5, 6] for more details. Note the special role that $t$ plays in the phase. All the geometric information is, as usual, encoded in the phase function $\phi$.

The main step in the proof is the construction of a parametrix for the propagator in the class of scattering-fibered Legendrians. One begins this process for $t$ near zero near the diagonal of $\mathbb{R}^{n} \times \mathbb{R}^{n}$ (and within the support of $\chi$ ). Here an ansatz for the propagator is the WKB expression

$$
t^{-n / 2} e^{i \Phi(z, w) / t} \sum_{j \geq 0} t^{j} a_{j}(z, w) .
$$

Solving for the phase in the usual way gives the eikonal equation

$$
\Phi=\frac{1}{2}\left|\nabla_{z} \Phi\right|^{2}
$$

for $\Phi$, which has a smooth solution $\Phi=\frac{1}{2} d_{g}(z, w)^{2}$, where $d_{g}$ is the distance with respect to the Riemannian metric $g$. This ansatz cannot be used for $z$ outside the injectivity radius of $w$, but a more complicated WKB-type ansatz

$$
t^{-n / 2-k / 2} \int_{\mathbb{R}^{k}} e^{i \Phi(z, w, v) / t} \sum_{j \geq 0} t^{j} a_{j}(z, w, v) d v
$$

remains valid for $z$ in any given compact set. The main problem is to write down a suitable ansatz encapsulating the asymptotics as $|z| \rightarrow \infty$.

In general, one solves a nonlinear first order PDE such as (10) by the method of characteristics. In this case the characteristics are given by curves

$$
s \mapsto\left(z=\gamma(s), \hat{\zeta}_{i}=g_{i j}\left(\gamma^{\prime}(s)\right)^{j}, t=0, \tau=s^{2} / 2\right)
$$

where $\gamma$ is the arc-length parametrized geodesic starting from $(w, \hat{\xi}) \in S_{w}^{*} \mathbb{R}^{n}$, and $\tau$ is the dual variable to $t$. The set of all such geodesics sweeps out a submanifold $L$ of $\mathbb{R}_{z}^{n} \times \mathbb{R}_{\zeta}^{n} \times \mathbb{R}_{\tau}$ which remains smooth beyond the injectivity radius. It is a Legendrian submanifold with respect to a naturally defined contact structure on $\mathbb{R}_{z}^{n} \times \mathbb{R}_{\zeta}^{n} \times \mathbb{R}_{\tau}$. If the projection of $L$ to the $z$ variable is a diffeomorphism, then one can write $\tau=\Phi(z)$ and this determines the phase function in (9). When this is not true, then one has to use the more complicated expression (11), where one requires $k$ extra variables in order to write $\tau$ as a function of $z$ and $v$ if the null space of the differential of the projection to $\mathbb{R}_{z}^{n}$ has dimension $k$. 
Along the geodesic, the form (31) of the metric implies (in the short-range case) that

$$
r(\gamma(s))=s+\Sigma(w, \hat{\xi})+O\left(s^{-1}\right), \quad \zeta(\gamma(s))=\frac{\gamma(s)}{|\gamma(s)|}+O\left(s^{-1}\right) .
$$

Hence $\tau(\gamma(s))=r^{2} / 2+r \Sigma(w, \hat{\xi})+O(1)$. Recall that we are interested in the phase of the propagator after an $e^{i r^{2} / 2 t}$ factor has been stripped out. Hence defining $\phi=\Phi-r^{2} / 2$, we find that $\phi \sim r \Sigma(x, \hat{\xi})$ at infinity. Since $\Phi$ (or more geometrically, the Legendre submanifold $L$ ) has good asymptotics at infinity, we can hope to write down an ansatz for the propagator which is accurate out to infinity and encodes the oscillations at spatial infinity as well as as $t \rightarrow 0$. In fact, this turns out to be possible in the class of fiberedscattering Legendre distributions and this gives a parametrix which differs from the true propagator by a kernel which is Schwartz in $z$ and $O\left(t^{\infty}\right)$, together with all its derivatives, as $t \rightarrow 0$.

Moreover, in the same way that $\Phi$ in the ansatz (11) is associated to a Legendre submanifold, so is $\phi(0, \cdot, \cdot, \cdot)$ in the expression (8) for $W_{t}$. This is proved essentially by symplectic reduction. The Legendre submanifold associated to $\phi$ at $r=\infty$ then turns out to be the graph of the transformation $S_{f}$, which is therefore a contact transformation.

The long-range case is not essentially different.

In the special case of flat $\mathbb{R}^{n}$, the sojourn time $\lambda$ along the geodesic emanating from $z$ in direction $\theta$ is

$$
\lim _{t \rightarrow \infty} t-|z+t \theta|=-z \cdot \theta
$$

Note that this is exactly the phase of $W_{t}$ in the Euclidean case, with no oscillatory integral necessary. This is typical of the following special geometric case: if for each $w$ and $\theta$ ranging over a pair of open sets, there exists a unique geodesic $\gamma$ with $\gamma(0)=w, \lim _{t \rightarrow \infty} \gamma(t) /|\gamma(t)|=\theta$, then we can let $S(w, \theta)$ denote the sojourn time along this geodesic (i.e. the function $\Sigma$ of (12), but parametrized by different variables). Then $S(w, \theta)$ parametrizes the Legendrian distribution, and we may write the propagator for $t \geq 0$ and $w \in U \Subset \mathbb{R}^{n}$ simply as

$$
t^{-n / 2} a\left(t, w, r^{-1}, \theta\right) e^{i S(w, \theta) / t}
$$

with $a$ a smooth function. The sojourn time $S(w, \theta)$ is closely related to a sojourn time defined on pairs of points in $S^{n-1}$ by Guillemin [4] in studying the high-frequency asymptotics of the scattering matrix.

\section{Propagation}

As a consequence of the characterization of $e^{-i \tilde{r}^{2} / 2 t} e^{-i t H}$ as a scattering FIO, where $\tilde{r}$ is given by (6) ( $\tilde{r}$ is just equal to $r$ in the short-range case), we can now state a propagation theorem, describing precisely when and where singularities can appear in $\mathbb{R}^{n}$ : 
Theorem 5. Let $\psi(t)$ be a solution to (11). Fix a time $t_{0}$. Let $(z, \hat{\zeta}) \in S^{*} \mathbb{R}^{n}$, and let

$$
(\theta, \xi)=S_{b}(z, \hat{\zeta}), \quad(\tilde{\theta}, \tilde{\xi})=S_{f}(z, \hat{\zeta})
$$

Then the following are equivalent:

$$
\begin{aligned}
(z, \hat{\zeta}) & \in \mathrm{WF}\left(\psi\left(t_{0}\right)\right) ; \\
\left(\theta, \frac{\xi}{t-t_{0}}\right) & \in \mathrm{WF}_{\mathrm{sc}}\left(e^{i \frac{\tilde{r}^{2}}{2\left(t-t_{0}\right)}} \psi(t)\right), t>t_{0} ; \\
\left(\tilde{\theta}, \frac{\tilde{\xi}}{t_{0}-t}\right) & \in \mathrm{WF}_{\mathrm{sc}}\left(e^{i \frac{\tilde{r}^{2}}{2\left(t-t_{0}\right)}} \psi(t)\right), t<t_{0} .
\end{aligned}
$$

This is a refinement of certain propagation results of [16], which were in terms of quadratic scattering wavefront set $\mathrm{WF}_{\mathrm{qsc}}$. In particular, one of the main results of [16] stated that if $\theta=\pi\left(S_{b}(z, \hat{\zeta})\right)$ denotes the backwards limit of the geodesic through $z, \hat{\zeta}$ then for $t>0$,

$$
(\theta,-\theta / 2 t) \notin \mathrm{WF}_{\mathrm{qsc}} \psi_{0} \Longrightarrow(z, \hat{\zeta}) \notin \mathrm{WF} \psi(t) .
$$

This clearly follows from Theorem 5 and the following wavefront set computations:

\section{Proposition 6.}

$$
(\theta, \xi) \in \mathrm{WF}_{\mathrm{qsc}} u \Longleftrightarrow(\theta, \xi-\alpha \theta / 2) \in \mathrm{WF}_{\mathrm{qsc}} e^{-i \alpha r^{2} / 2} u .
$$

Proposition 7. If $(\theta, 0) \notin \mathrm{WF}_{\mathrm{qsc}} u$ then $(\theta, \xi) \notin \mathrm{WF}_{\mathrm{sc}} u$ for any finite $\xi$.

The proofs of Propositions 6 17 which are not difficult, are best carried out using the more sophisticated definitions of wavefront set, involving associated calculi of pseudodifferential operators.

\section{EXAMPLES}

We conclude with a pair of examples which exhibit some extremes of behavior which solutions of (11) may exhibit.

First, we consider the Schrödinger equation in flat $\mathbb{R}^{n}$ with $V=0$. Let

$$
\psi_{0}(z)=(-2 \pi i)^{-1 / 2} e^{-i z_{1}^{2} / 2} ;
$$

note that this is just the one-dimensional fundamental solution, evaluated at time $t=-1$, and extended to be constant in the variables $z_{2}, \ldots, z_{n}$. The quadratic scattering wavefront set of $\psi_{0}$ satisfies the hypotheses of (13) over two points in $S_{\hat{z}}^{n-1}$, given by $( \pm 1,0, \ldots, 0)$, hence (13) guarantees that the wavefront set of $\psi(t)$ is confined at most to $\{t=1, \zeta=( \pm 1,0, \ldots, 0)\}$. On the other hand, we can compute exactly: $\psi(1, z)=\delta\left(z_{1}\right)$. Hence two points in the qsc wavefront set are able to produce an entire hyperplane of 
singularities. By contrast, Theorem [5 gives a more satisfactory picture of the propagation phenomenon: we have

$$
e^{i r^{2} / 2} \psi_{0}=e^{i\left(z^{\prime}\right)^{2} / 2}
$$

(where $z^{\prime}=\left(z_{2}, \ldots, z_{n}\right)$ ). Since the Fourier transform of this function is just

$$
\frac{1}{i^{(n-1) / 2} \sqrt{2 \pi}} e^{-i\left(\zeta^{\prime}\right)^{2} / 2} \delta\left(\zeta_{1}\right),
$$

we have

$$
\mathrm{WF}_{\mathrm{sc}}\left(e^{i r^{2} / 2} \psi_{0}\right)=\left\{\left(( \pm 1,0, \ldots, 0),\left(0, \zeta^{\prime}\right)\right): \zeta^{\prime} \in \mathbb{R}^{n-1}\right\}
$$

This is pair of hyperplanes over two points in $S_{\hat{\zeta}}^{n-1}$ which maps diffeomorphically to $\operatorname{WF} \psi(1, z)$ according to the sojourn relation.

Another extreme case for the results discussed above occurs if we work on $\mathbb{R}^{1}$ with $V=0$, and take

$$
\psi_{0}(z)=e^{-i z^{2} / 2} \operatorname{Ai}(z) .
$$

The quadratic-scattering wavefront set of $\psi_{0}$ is just $(-1,1 / 2)$ (Intuitively speaking, the factor of Ai kills the wavefront set at $\hat{z}=+1$ owing to its exponential decay in that direction, but has no effect on the wavefront set of the $e^{-i z^{2} / 2}$ factor at $\hat{z}=-1$ owing to its slower oscillation.) Hence (13) permits wavefront set only at $t=1$, in the direction $\zeta=+1$. On the other hand, we may compute exactly to find that

$$
\psi(z, 1)=(-2 \pi i)^{1 / 2} e^{i\left(\frac{z^{3}}{3}+\frac{z^{2}}{2}\right)} \in \mathcal{C}^{\infty}(\mathbb{R}) ;
$$

hence no wavefront set appears after all. This situation is accounted for in the results of [16] by the use of a wavefront set that is uniform in time, and in terms of which this solution is singular everywhere on $\mathbb{R}$ at $t=1$. In terms of Theorem [5] which is about the wavefront set of $\psi(\cdot, t)$ for each fixed $t$, the explanation is as follows: both $e^{i r^{2} / 2} \psi_{0}$ and $\psi(z, 1)$ have scattering wavefront set only at the corner $S^{0} \times S^{0}$, alluded to in the remark following Definition 2 As there are no points $(\hat{z}, \zeta)$ in $\mathrm{WF}_{\mathrm{sc}}\left(e^{i r^{2} / 2} \psi_{0}\right)$ with $\zeta$ finite, Theorem 5 asserts that $\psi(1, z)$ is smooth.

\section{REFERENCES}

[1] J. Brüning and V.W. Guillemin (Editors), Fourier integral operators, Springer-Verlag, Berlin, Heidelberg, New York, Tokyo, 1994.

[2] John D. Dollard, Quantum-mechanical scattering theory for short-range and Coulomb interactions, Rocky Mountain J. Math. 1, 1971, 5 -88. MR 42 \#5561

[3] Daisuke Fujiwara, Remarks on convergence of the Feynman path integrals, Duke Math. J. 47 (1980), no. 3, 559-600. MR 83c:81030

[4] V. Guillemin, Sojourn times and asymptotic properties of the scattering matrix, Proceedings of the Oji Seminar on Algebraic Analysis and the RIMS Symposium on Algebraic Analysis (Kyoto Univ., Kyoto, 1976), vol. 12, 1976/77 supplement, pp. 6988. MR 56 \#6759 
[5] A. Hassell and A. Vasy, The resolvent for Laplace-type operators on asymptotically conic spaces, Ann. Inst. Fourier (Grenoble) 51 (2001), no. 5, 1299-1346. MR 2002i: 58037

[6] Andrew Hassell and András Vasy, The spectral projections and the resolvent for scattering metrics, J. Anal. Math. 79 (1999), 241-298. MR 2001d:58034

[7] Andrew Hassell and Jared Wunsch, The Schrödinger propagator for scattering metrics, Preprint, January 2003.

[8] L. Hörmander, Fourier integral operators, I, Acta Math. 127 (1971), 79-183, See also [1.

[9] L. Kapitanski and Yu. Safarov, A parametrix for the nonstationary Schrödinger equation, Differential operators and spectral theory, Amer. Math. Soc. Transl. Ser. 2, vol. 189, Amer. Math. Soc., Providence, RI, 1999, pp. 139-148. MR 2000j:35236

[10] Lev Kapitanski and Yuri Safarov, Dispersive smoothing for Schrödinger equations, Math. Res. Lett. 3 (1996), no. 1, 77-91. MR 97g:35030

[11] Richard Melrose and Maciej Zworski, Scattering metrics and geodesic flow at infinity, Invent. Math. 124 (1996), no. 1-3, 389-436. MR 96k:58230

[12] Richard B. Melrose, Spectral and scattering theory for the Laplacian on asymptotically Euclidian spaces, Spectral and scattering theory (Sanda, 1992), Dekker, New York, 1994, pp. 85-130. MR 95k:58168

[13] Luc Robbiano and Claude Zuily, Analytic theory for the quadratic scattering wave front set and application to the Schrödinger equation, Astérisque (2002), no. 283, vi+128. MR 1958605

[14] Leonard Schiff, Quantum Mechanics, McGraw-Hill, 1968, Singapore.

[15] François Trèves, Parametrices for a class of Schrödinger equations, Comm. Pure Appl. Math. 48 (1995), no. 1, 13-78. MR 95m:35050

[16] Jared Wunsch, Propagation of singularities and growth for Schrödinger operators, Duke Math. J. 98 (1999), no. 1, 137-186. MR 2000h:58054

[17] Kenji Yajima, Smoothness and non-smoothness of the fundamental solution of time dependent Schrödinger equations, Comm. Math. Phys. 181 (1996), no. 3, 605-629. MR 97j:35001

[18] Steven Zelditch, personal communication.

[19] - Reconstruction of singularities for solutions of Schrödinger's equation, Comm. Math. Phys. 90 (1983), no. 1, 1-26. MR 85d:81029 